\title{
MicroRNA- 126 Level Increases During Exercise Rehabilitation of Heart Failure with a Preserved Ejection Fraction
}

\author{
Dong Jin \\ Xiu-Yun Yang \\ Jie-Sheng Wang
}

Geriatrics Department, Tongde Hospital of Zhejiang Province, Hangzhou, Zhejiang Province, People's Republic of China
Correspondence: Dong Jin Geriatrics Department, Tongde Hospital of Zhejiang Province, Hangzhou, Zhejiang Province, 3100I2, People's Republic of China

Tel +86-13958I33668

Email2168256719@qq.com
Objective: To evaluate the changes of plasma levels of miR-126 in heart failure with a preserved ejection fraction (HFpEF) patients undergoing an exercise rehabilitation intervention.

Methods: miR-126 levels in plasma were compared between $60 \mathrm{HFpEF}$ patients and 30 healthy volunteers. HFpEF patients underwent exercise rehabilitation for 12 weeks. Before and after rehabilitation, indicators of cardiac function, exercise tolerance, quality of life scores and miR-126 levels were measured and compared. Correlations between plasma levels of miR-126 and HFpEF were evaluated.

Results: The plasma levels of miR-126 in HFpEF patients were lower than those in healthy volunteers and increased significantly after exercise rehabilitation. HFpEF patients also showed significantly better cardiac function, exercise tolerance, and quality of life after rehabilitation. The results of Pearson correlation analysis and multiple linear regression showed that miR-126 levels were positively correlated with peak oxygen consumption (peak $\mathrm{VO}_{2}$ ) and metabolic equivalents (METs), and inversely associated with score on the Minnesota Living with Heart Failure Questionnaire (MLHF) as well as plasma N-terminal pro-B-type natriuretic peptide (NT-proBNP) levels.

Conclusion: miR-126 levels are low expressed in plasma among HFpEF patients. Effective exercise rehabilitation in HFpEF patients may positively impact the plasma level of miR126 , which is probably associated with the restoration of cardiac function, exercise tolerance and quality of life. miR-126 may be a potential biomarker for evaluating the efficacy of exercise rehabilitation for HFpEF patients.

Keywords: HFpEF, miR-126, exercise rehabilitation

\section{Introduction}

The pathophysiologic mechanism of HFpEF has not been fully elucidated. Recently, some scholars have argued that endothelial injury plays a major role in the development of HFpEF. $^{1-3}$ The recovery of endothelial dysfunction is primarily correlated with rehabilitation in HFpEF patients. ${ }^{4,5}$ microRNAs are tiny non-coding RNA molecules make up of approximately 23-25 nucleotides. They regulate the gene expressions involved in various cellular processes. Recently, plasma miRNAs have been reported to be sensitive and specific biomarkers of various tissue injuries and pathological conditions. ${ }^{5}$ microRNA-126 (miR-126) is specifically and primarily expressed by the vascular endothelium. A number of reports have shown that miR-126 levels are lower in patients with endothelial injury or dysfunction, ${ }^{4,5}$ while higher expression of miR-126 
positively impacts the restoration process of the impaired endothelium. ${ }^{4-6}$ Changes in miR-126 expression greatly affect endothelial function by managing the pathophysiological processes of cardiovascular development, preventing vascular inflammation and retaining neovascularization. ${ }^{7}$ Therefore, miR-126 levels are closely related to endothelial dysfunction. Alterations in miR-126 expression probably reflect the pathological evolution of endothelial dysfunction in patients with HFpEF. $^{8-10}$ In this report, we assumed that the miR-126 levels of HFpEF patients were lower than those of healthy volunteers. After exercise rehabilitation, increased plasma level of miR-126 was thought to be actively promoted, which may have been related to the improvements in clinical condition among HFpEF patients.

According to the experimental design, the plasma levels of miR-126 among HFpEF patients were measured and compared with those of healthy volunteers before rehabilitation. Before and after 12 weeks of exercise training, the changes in the plasma levels of miR-126 in HFpEF patients, as well as clinical parameters, were observed and compared. Correlation analysis between miR-126 levels and clinical indices of HFpEF was performed for preliminary assessment of the clinical significance of miR-126 levels in HFpEF patients.

\section{Materials and Methods}

\section{Settings and Patients}

Initially, $74 \mathrm{HFpEF}$ patients were enrolled for eligibility assessment. A total of $65 \mathrm{HFpEF}$ patients who met the inclusion and exclusion criteria were recruited. 60 patients finished 12 weeks of exercise training, and their data were collected for the final statistical analysis. Baseline characteristics of these patients are displayed in Table 1. In addition, our study also included 30 healthy individuals with normal heart function as a control group (13 men and 17 women, mean age: $66.83 \pm 9.76$ years). Normal healthy people were gathered from the medical examination center of our hospital as the control group. The plasma miR-126 levels of 30 healthy volunteers were tested and compared with those of HFpEF patients. The recruitment procedures are presented in Figure 1.

The inclusion criteria for this trial were as follows: (1) characteristic signs and symptoms of heart failure and preserved left ventricular ejection fraction (LVEF) $\geq$ $50 \%$; (2) 55-70 years old; (3) NYHA class II or class III; and (4) clinically stable without significant deterioration of cardiac condition for at least 4 weeks.
Table I Baseline Characteristics of $60 \mathrm{HFpEF}$ Patients in This Study

\begin{tabular}{|l|c|}
\hline & HFpEF Patients (n=60) \\
\hline Age & $68.3 \pm 7.8$ \\
Sex (male/female, $n)$ & $27 / 33$ \\
BMI $\left(\mathrm{Kg} / \mathrm{m}^{2}\right)$ & $23 \pm 6$ \\
SBP $(\mathrm{mmHg})$ & $143 \pm 15$ \\
DBP (mmHg) & $81 \pm 14$ \\
NYHA (II/III, $n$ ) & $29 / 31$ \\
Coronary heart disease history (n) & 39 \\
Diabetes history (n) & 45 \\
Hypertension history (n) & 38 \\
Smoker (n) & 11 \\
Dyslipidemia (n) & 31 \\
\hline Medication & \\
Nitrates (n) & 24 \\
Statatin (n) & 35 \\
Aspirin (n) & 27 \\
Clopidogrel (n) & 33 \\
ACEl or ARB (n) & 46 \\
b-blockers (n) & 35 \\
Aldosterone inhibitors (n) & 42 \\
Thiazide diuretics (n) & 23 \\
Loop diuretics (n) & $1 \mathrm{I}$ \\
Insulin (n) & 27 \\
\hline
\end{tabular}

Abbreviations: BMI, body mass index; SBP, systolic blood pressure; DBP, diastolic blood pressure; HFpEF, heart failure with preserved ejection fraction; NYHA, New York heart association; ACEI/ARB, angiotensin enzyme inhibitor/ angiotensin receptor blocker; b-blockers, b-adrenergic Receptor Antagonism.

The following patients were excluded from this study: (1) $<55$ or $>70$ years old; (2) complicated with acute myocarditis, unstable angina pectoris, or acute myocardial infarction in the past 4 weeks; (3) valvular heart disease, congenital heart disease, or thyroid disease; (4) complicated by complete atrioventricular block, pericarditis, or frequent ventricular premature in the 28 days before the test; (5) complicated by electrolyte disturbances, pulmonary embolism, renal failure, liver dysfunction, or serious infections; (6) current pregnancy or lactation; and (7) undergoing psychiatric treatment.

This study followed the Ethics Guidelines of the 1975 Declaration of Helsinki. The experimental scheme was examined and approved by the Ethics Committee of Tongde Hospital of Zhejiang Province, China. Each participant voluntarily joined this study and signed an informed consent form before the test.

\section{Procedures for Exercise Rehabilitation}

Rehabilitation instruction was provided for each patient with HFpEF before the test. The protocol for 12 weeks 


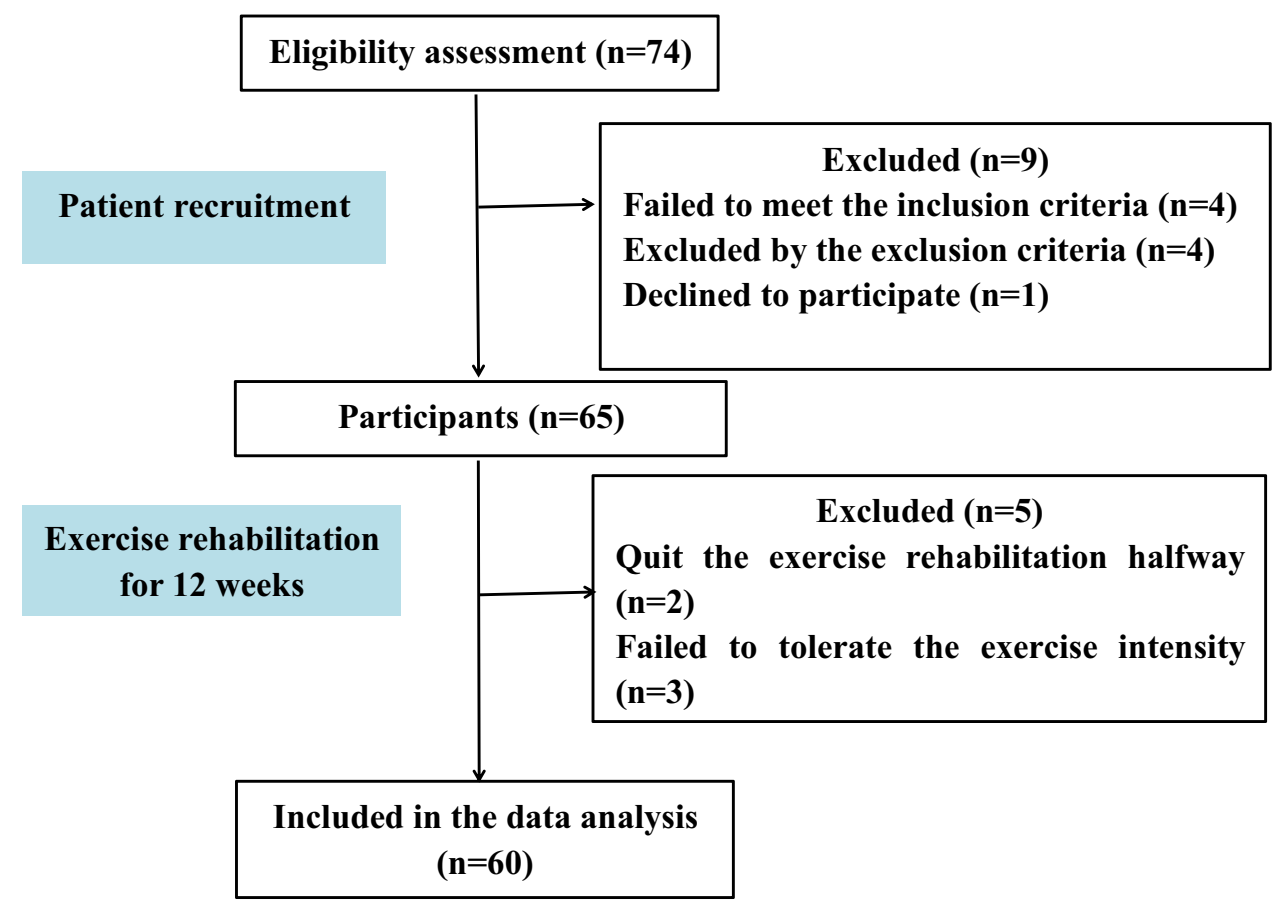

Figure I Flowchart of HFpEF patient enrollment.

of cardiac rehabilitation included aerobic exercise and resistance exercise. Aerobic exercise was performed 3 times per week based on the value of metabolic equivalents (METs) at the anaerobic threshold in the cardiopulmonary exercise test. The patients with 5-6 METs performed treadmill exercise with a power of 100 watts. Those with more than 6 METs adjusted their treadmill power to the target heart rate [(maximum heart rate resting heart rate) $\times 60 \%+$ resting heart rate]. Each exercise consisted of a 5-minute warm-up, 20 minutes of exercise training and 5 minutes of relaxation. Resistance exercise was performed with an elastic band 3 times a week. After 5 minutes of warm-up, 8-12 muscle groups were trained for 20 minutes and relaxed for 5 minutes each. Medical staff supervised the whole process of exercise rehabilitation to ensure the safety of the HFpEF patients. Exercise training was stopped, and relevant treatment was employed immediately if the following dangerous situations occurred: (1) the heart rate exceeded the target heart rate; (2) the systolic blood pressure increased over $30 \mathrm{mmHg}$ or decreased over $10 \mathrm{mmHg}$; (3) the patient had unbearable symptoms such as chest tightness, sweating, dizziness, and dyspnea; (4) exercise electrocardiogram showed ST segment depression $>0.1 \mathrm{MV}$ or ST segment elevation $>0.1 \mathrm{MV}$; or (5) serious arrhythmia was detected during exercise.
At baseline and 12 weeks after exercise rehabilitation, the patients were subjected to cardiopulmonary exercise tests, echocardiography examinations, quality of life assessments, 6-minute walk tests (6MWT) and NT-proBNP detection for clinical efficacy evaluation. The indicators of exercise tolerance were measured through the cardiopulmonary exercise test and 6MWT, including peak $\mathrm{VO}_{2}$, $\mathrm{VE} / \mathrm{VCO}_{2}$ slop, $\mathrm{VO}_{2} \mathrm{AT}$, METs and walking distance in the 6MWT. The parameters of cardiac function were primarily tested by echocardiography, including left ventricular ejection fraction (LVEF), cardiac index (CI), the ratio of early diastolic mitral flow velocity to early diastolic mitral annular velocity (E/e'), left atrial volume index (LAVI), and left ventricular muscle mass index (LVMI). The Minnesota Living with Heart Failure Questionnaire (MLHF) score was used to assess the quality of life of HFpEF patients.

The NT-proBNP level was detected by immunoassay. Venous blood $(5 \mathrm{~mL})$ was collected from every patient, put into an EDTA anticoagulant tube, and centrifuged at 3000 revolutions per minute for 20 minutes to obtain the isolated plasma. The level of NT-proBNP expression in the isolated plasma was detected by fluorescence immunoassay. The Von Willebrand Factor (vWF) level was detected via ELISA using the following method: the collected venous blood was treated following the instructions of the ELISA kit (Sun Biotechnologies Co., Ltd., China), and then, the plasma 
vWF content was detected in accordance with the instructions of the ELISA kit.

\section{The Measurement of microRNA- 126 Expression Levels}

Fasting blood samples were collected from elbow vein early in the morning. Samples of venous blood $(5 \mathrm{~mL})$ were acquired in vacuum-dried tubes with RNA-free enzymes. Then, the blood samples were isolated by centrifugal separation at $3000 \mathrm{~g}$ for $10 \mathrm{~min}$ at room temperature. Serum was transported to nuclease-free Eppendorf tubes and preserved at $-80^{\circ} \mathrm{C}$ until microRNA could be isolated.

In Brief, microRNA was isolated from stored serum samples by performing a microRNA easy kit (Aidlab Biotechnologies Co., Ltd., China) and conforming to the manufacturer's instructions. ${ }^{11}$ The resultant microRNA was maintained for quantitative real-time PCR (qRT-PCR). The miR-126 PCR primer was obtained from Ribobio Co., Ltd (China). The primers of miR-126 used in this study were as followed: 5-TCGTACCGTGAGTAATAATGCG-3. Following the manufacturer's instructions for qRT-PCR, complementary DNA (cDNA) was synthesized from $1 \mathrm{ug}$ of total RNA by reverse transcription in the GoScriptt Reverse Transcription System (Promega Co., Ltd., USA). In brief, the reaction procedures included incubation at $25^{\circ} \mathrm{C}$ for 5 minutes, $42^{\circ} \mathrm{C}$ for 60 minutes, and $70^{\circ} \mathrm{C}$ for 15 minutes, and the samples were then kept at $20^{\circ} \mathrm{C}$ until further study. QT- PCR was carried out under the following conditions by GoTaq qPCR Master Mix (Promega Co., Ltd.): denaturation at $95^{\circ} \mathrm{C}$ for 2 minutes, followed by 40 cycles of annealing at $95^{\circ} \mathrm{C}$ for 15 seconds and extension at $60^{\circ} \mathrm{C}$ for 60 seconds.

The levels of miR-126 were determined by the $2-\Delta \Delta \mathrm{Ct}$ method. ${ }^{12}$ The primers used UniSp6 as the endogenous reference. The comparative expression level of miR-126 is presented as miR-126 relative to an internal control gene and named $\Delta \mathrm{Ct}$. Subsequently, mathematical formulas were used to calculate $\Delta \mathrm{Ct}$ and $\Delta \Delta \mathrm{Ct}: \Delta \mathrm{Ct}=\mathrm{Ct}_{\text {miR-126 }}$ $\mathrm{Ct}_{\text {UniSp6 }}$ and $\Delta \Delta \mathrm{Ct}=\Delta \mathrm{Ct}_{\text {case }}-\Delta \mathrm{Ct}_{\text {control }}$.

\section{Statistical Analysis}

Statistical analysis was performed with SPSS 18.0 software (SPSS Inc., USA). Data are shown as mean \pm standard deviation. Before and after rehabilitation, comparisons of clinical indices were performed with paired t-tests. $\mathrm{P}<0.05$ was considered statistically significant. Pearson's correlation analyses were carried out between different indicators and
miR-126 levels. After adjusting for differences in confounding factors such as diabetes, age and drug use, multiple linear regression analyses were carried out to appraise independent associations between miR-126 and clinical parameters.

\section{Results}

After 12 weeks of exercise rehabilitation, HFpEF patients showed significantly increased peak $\mathrm{VO}_{2}, \mathrm{VO}_{2} \mathrm{AT}, 6 \mathrm{MWT}$, METs as well as vWF levels. Obvious decreases were found in $\mathrm{VE} / \mathrm{VCO}_{2}$ slop, E/e, LAVI and NT-proBNP levels. After rehabilitation, HFpEF patients also had a better life of quality by decreased MLHF scores (Table 2).

Compared with those of the healthy volunteers, the plasma miR-126 levels of HFpEF patients were significantly lower. At the end of exercise rehabilitation, the miR-126 levels of plasma among HFpEF patients increased significantly than the baseline, although they were still lower than those of the controls (Table 3).

Pearson correlation analysis initially revealed relationship between clinical indices and the expression levels of miR-126 (Table 4). miR-126 levels were positively associated with peak $\mathrm{VO}_{2}$, METs, $\mathrm{VO}_{2} \mathrm{AT}$ and LVEF. miR-126 levels were negatively associated with NT-proBNP levels, LAVI, E/e', the MLHF score and $\mathrm{VE} / \mathrm{VCO}_{2}$ slop. After adjusting for confounding factors and counting on the results of multiple linear regression analyses (Table 5), miR-126 levels were positively related to peak $\mathrm{VO}_{2}$ and METs but negatively related to MLHF scores and NTproBNP levels (Figure 2).

\section{Discussion}

HFpEF accounts for almost $50 \%$ of all heart failure admissions. Accumulating evidence has demonstrated that microRNAs are closely associated with the development and prognosis of heart failure, but the underlying mechanisms remain poorly understood. ${ }^{11-13}$ miR-126 is a proangiogenic microRNA that is strongly expressed in the heart endothelium. It plays a key role in retaining endothelial function and determining endothelial homeostasis by inducing angiogenesis. ${ }^{13}$ The elevation of miR126 expression can promote restoration of the damaged endothelium. ${ }^{13}$ Before testing, we found that the average levels of miR-126 in HFpEF patients were substantially lower than those in healthy volunteers. After exercise, the levels of miR-126 in HFpEF patients increased significantly but were still lower than those in healthy controls. Lower levels of miR-126 imply that the recovery functions of the endothelium are impaired in HFpEF patients. 
Table 2 Comparison of Data Obtained Before and After Rehabilitation

\begin{tabular}{|l|c|c|c|}
\hline & Before Rehabilitation $\mathbf{( n = 6 0 )}$ & After Rehabilitation $\mathbf{( n = 5 0 )}$ & $\boldsymbol{P}$ \\
\hline SDP $(\mathrm{mmHg})$ & $143(15)$ & $138(17)$ & 0.73 \\
DBP $(\mathrm{mmHg})$ & $81(14)$ & $76(12)$ & 0.79 \\
NT-proBNP $(\mathrm{ng} / \mathrm{mL})$ & $1411(293)$ & $956(143)$ & 0.01 \\
6MWT $(\mathrm{m})$ & $252(82)$ & $434(65)$ & 0.01 \\
PeakVO $(\mathrm{mL} / \mathrm{min} / \mathrm{kg})$ & $13.5(6.7)$ & $16.6(7.2)$ & 0.02 \\
VO ${ }_{2}$ AT $(\mathrm{mL} / \mathrm{min} / \mathrm{kg})$ & $9.1(3.6)$ & $12.1(4.2)$ & 0.03 \\
METs & $5.42(1.61)$ & $6.67(1.89)$ & 0.02 \\
VE/VCO ${ }_{2} \mathrm{slop}$ & $37.7(5.1)$ & $30.7(5.5)$ & 0.01 \\
LVEF $(\%)$ & $59.0(8.3)$ & $61.1(7.6)$ & 0.32 \\
Cl $\left(\mathrm{L} / \mathrm{min} / \mathrm{m}^{2}\right)$ & $2.8(0.5)$ & $3.0(0.6)$ & 0.69 \\
LAVI $\left(\mathrm{mL} / \mathrm{m}^{2}\right)$ & $36.7(5.5)$ & $31.5(6.5)$ & 0.04 \\
LVMI $\left(\mathrm{mg} / \mathrm{m}^{2}\right)$ & $134.0(33.3)$ & $126.0(27.3)$ & 0.13 \\
E/e' & $16.4(5.3)$ & $13.1(4.6)$ & 0.04 \\
MLHF & $47(13)$ & $56(14)$ & 0.02 \\
vWF $(\mathrm{ng} / \mathrm{L})$ & $13.13(3.47)$ & $18.56(5.14)$ & 0.01 \\
\hline
\end{tabular}

Abbreviations: SBP, systolic blood pressure; DBP, diastolic blood pressure; NT-proBNP, N-terminal prohormone brain natriuretic peptide; 6MWT, 6-minute walk test; LVEF, left ventricular ejection fraction; peakVO 2 , peak oxygen uptake; $\mathrm{VO}_{2} \mathrm{AT}$, anaerobic threshold; $M E T s$, metabolic equivalents; $V E / V C O{ }_{2}$ slop, the slope of the relation between ventilation and carbon dioxide production; LVMI, left ventricular mass index; LAVI, left atrial volume index; Cl, cardiac output index; E/e', diastolic mitral flow velocity and diastolic mitral annulus velocity ratio; MLHF, the Minnesota Living With Heart Failure score; vWF, Von Willebrand Factor.

Previously, it was reported that there was a decline in the plasma concentrations of miR-126 in patients with heart failure. ${ }^{14}$ In heart muscle of female Wistar rats, the apoptosis of vascular endothelial cell was increased by miR126 inhibition and obviously rescued by miR-126 overexpression. ${ }^{15}$ Knockdown of the miR-126 gene in an animal model of heart failure may result in diminished vascular integrity and decreased density of the microvasculature. ${ }^{16}$ Generally, reduced plasma levels of miR-126 inhibit endothelial cell migration during the progression of vessel growth, differentiation, and organization, leading to accelerated pathological development of heart failure. ${ }^{17}$ Hence, reduced miR-126 level in plasma may be related to the clinical conditions of patients with chronic heart failure, and it could be effective in the diagnosis of chronic heart failure. ${ }^{17}$ Interestingly, on the basis of the Pearson's correlation and multiple linear regression analyses in this report, the miR-126 level of

Table 3 Comparisons of miR-126 Levels in Plasma Between HFpEF Patients and Healthy Volunteers

\begin{tabular}{|l|c|c|}
\hline & \multicolumn{2}{|c|}{ miR-I 26 Level } \\
\cline { 2 - 3 } & At Baseline & After Rehabilitation \\
\hline HFpEF patients $(n=60)$ & $3.09 \pm 0.56$ & $4.35 \pm 0.79^{\#}$ \\
Healthy volunteers $(n=30)$ & $9.56 \pm 1.87^{* \Delta}$ & \\
\hline
\end{tabular}

Notes: $* \mathrm{P}=0.0$ I, compared to $\mathrm{HFpEF}$ patients at baseline; ${ }^{\triangle} \mathrm{P}=0.01$, compared to HFPEF patients after rehabilitation; ${ }^{\#} \mathrm{P}=0.02$, compared to baseline. plasma was also proven to be associated with some important indices of cardiac function in HFpEF patients, such as peak $\mathrm{VO}_{2}$, METs, the MLHF score and NT-proBNP levels. It suggests that decreased miR-126 expression in plasma may partly reflect the worse clinical condition of patients with HFpEF compared to normal controls. Similarly, lower plasma levels of miR-126 have been reported to be

Table 4 Pearson Correlation Analysis Between miR-I26 Expression Levels and Clinical Indicators in HFpEF Patients

\begin{tabular}{|c|c|c|}
\hline Factors & $\mathbf{R}$ & $P$ \\
\hline NT-proBNP & $-0.415^{*}$ & 0.01 \\
\hline 6MWT & 0.217 & 0.27 \\
\hline PeakVO & $0.531 *$ & 0.009 \\
\hline METs & $0.498^{*}$ & 0.01 \\
\hline $\mathrm{VO}_{2} \mathrm{AT}$ & $0.46 I^{*}$ & 0.007 \\
\hline $\mathrm{VE} / \mathrm{VCO}_{2}$ slop & $-0.27 I^{*}$ & 0.03 \\
\hline LVEF & $0.476 *$ & 0.02 \\
\hline $\mathrm{Cl}$ & 0.187 & $0.4 I$ \\
\hline LAVI & $-0.459 *$ & 0.01 \\
\hline LVMI & -0.134 & 0.43 \\
\hline$E / e^{\prime}$ & $-0.339 *$ & 0.01 \\
\hline MLHF & $-0.497^{*}$ & 0.007 \\
\hline
\end{tabular}

Note: $* \mathrm{P}<0.05$.

Abbreviations: NT-proBNP, N-terminal prohormone brain natriuretic peptide; 6MWT, 6-minute walk test; peakVO 2 , peak oxygen uptake; METs, metabolic equivalents; $\mathrm{VO}_{2} \mathrm{AT}$, anaerobic threshold; $\mathrm{VE} / \mathrm{VCO}_{2}$ slop, the slope of the relation between ventilation and carbon dioxide production; $\mathrm{Cl}$, cardiac output index; LVEF, left ventricular ejection fraction LVMI, left ventricular mass index; LAVI, left atrial volume index; E/e', diastolic mitral flow velocity and diastolic mitral annulus velocity ratio; MLHF, the Minnesota Living With Heart Failure score. 
Table 5 Results of Multiple Linear Regression Analysis

\begin{tabular}{|l|c|c|c|c|c|}
\hline Factors & B & Standard Error & $\boldsymbol{P}$ & Odds Ratio & 95\% Confidence Interval \\
\hline NT-proBNP & -0.879 & 0.261 & 0.018 & -2.513 & $-1.761 \sim-3.206$ \\
PeakVO $_{2}$ & 1.231 & 0.256 & 0.011 & 2.237 & $1.937-2.634$ \\
METs & 1.513 & 0.315 & 0.021 & 2.076 & $1.678-2.913$ \\
MLHF & 1.013 & 0.165 & 0.023 & 1.537 & $1.236-1.918$ \\
\hline
\end{tabular}

Abbreviations: NT-proBNP, N-terminal prohormone brain natriuretic peptide; peak $\mathrm{VO}_{2}$, peak oxygen uptake; METs, metabolic equivalents; MLHF, Minnesota Living with Heart Failure questionary.

correlated with left ventricular function, age, and NTproBNP levels in heart failure patients. ${ }^{14,18}$ Thus, the decreased plasma levels of miR-126 in HFpEF patients probably represent worse recovery of endothelial function and declined clinical function.

Another important finding in this study was that the plasma levels of miR-126 expression increased after exercise rehabilitation. After 12 weeks of exercise, we found that the plasma levels of miR-126 among HFpEF patients increased significantly compared with those observed at baseline, followed by better indices of cardiac function, exercise tolerance and MLHF scores. This result conformed to our original presumption that the restorative increase in miR-126 levels may reflect effective rehabilitation in $\mathrm{HFpEF}$ patients.

\section{Correlation of miR-126}

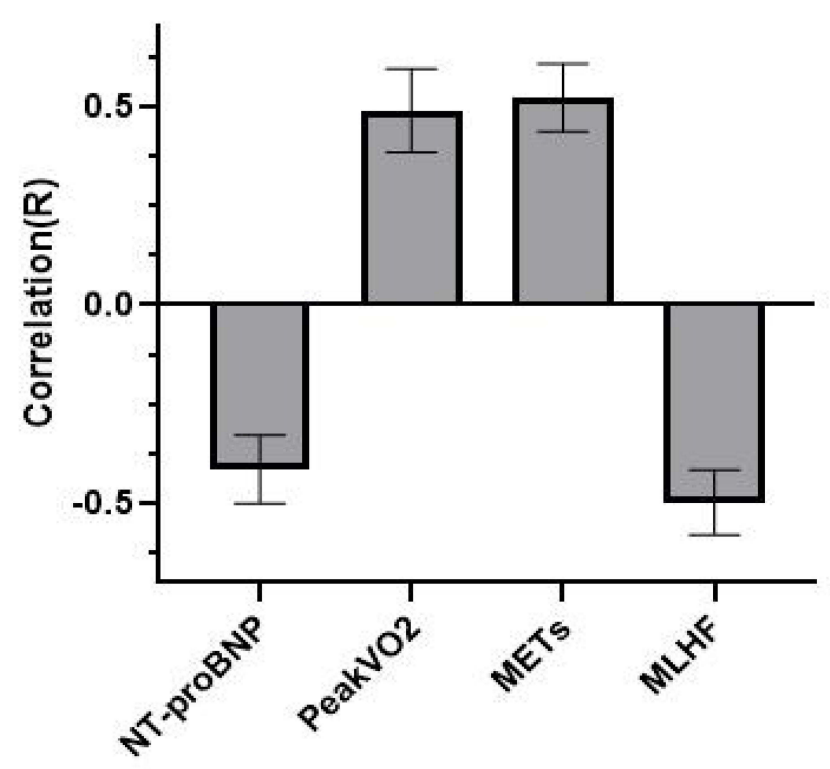

Figure 2 Correlation between miR-I26 plasma expression levels and clinical indicators in HFpEF patients.

Abbreviations: NT-proBNP, N-terminal prohormone brain natriuretic peptide; peakVO2, peak oxygen uptake; METs, metabolic equivalents; MLHF, the Minnesota Living With Heart Failure score.
Dastah et al $^{19}$ found that exercise training made cardioprotective effects on the heart tissue of diabetic rats by upregulating miR-126 and angiogenic signaling pathways. Increased expression of plasma miR-126 is associated with the activation of vascular endothelial growth factor (VEGF). ${ }^{20}$ miR-126 has the best known role in control of the formation and maintenance of angiogenesis and vascular integrity, it can directly inhibit two gene targets of the VEGF pathway. ${ }^{20}$ The two validated gene targets that are related to angiogenic signaling as negative regulators of VEGF signaling pathways: Sprouty-related protein 1 (Spred-1), an intracellular suppressor of the Ras/MAPK pathway, ${ }^{21}$ and phosphoinositol-3 kinase regulatory subunit 2 (PI3KR2), which negatively governs the activity of PIK3/Akt/eNOS pathways. ${ }^{20,21}$ Aerobic rehabilitation increased the expression of miR-126 in plasma which is probably associated with exercise-induced cardiac angiogenesis, by indirect regulation of the VEGF pathway and direct regulation of its targets that converged in an increase in angiogenic pathways, such as MAPK and PI3K/Akt/eNOS. ${ }^{22}$ Thereby, the growing miR126 after exercise in HFpEF patients may suggest that miR126 is a potential therapeutic target of exercise rehabilitation in pathological conditions of HFpEF.

Last but not the least important, the levels of plasma miR-126, were shown to be associated with plasma level of NT-proBNP, peakVO 2 , METs and MLHF score in this study (Figure 2). This result suggests the increasing degree of miR-126 plasma level may be helpful for evaluating exercise efficacy among HFpEF patients in the rehabilitation phase. According to the precious report, heart failure patients with improved plasma miR-126 levels also showed better exercise performance and lower B-type natriuretic peptide (BNP) levels. ${ }^{7,23}$ In two independent cohorts of 2203 subjects, increased circulating miR-126 was also related to reduced risk of death and rehospitalization in patients with chronic heart failure. ${ }^{7}$ Moreover, higher levels of miR-126 were related to lower rates of rehospitalization and death in the year after the initial 
admission for acute heart failure and decreased risk of cardiovascular events in patients with chronic heart failure of ischemic etiology for 2 years. ${ }^{12}$ Consequently, heart failure patients with higher levels of miR-126 probably have better clinical status and prognosis than those with lower levels of miR-126. miR-126 may serve as a potential biomarker for evaluating the curative effect and prognosis among heart failure patients.

In conclusion, the plasma level of miR-126 decreased in HFpEF patients. Decreasing miR-126 levels may underlie variations in regressive cardiac function among $\mathrm{HFpEF}$ patients. The levels of miR-126 expression can increase again after exercise rehabilitation, initiating the process of the angiogenesis in HFpEF patients. Collectively, the miR126 expression level, may serve as a potential indicator for evaluating the effects of rehabilitation among HFpEF patients. However, there are some potential limitations of this report. The experimental samples were not large, which may affect the clinical relevance of plasma miR-126 expression. Future studies with a larger number of patients from multiple centers are necessary to confirm our results.

\section{Data Sharing Statement}

All data in this article, including 5 tables and 2 figures, can be published and shared for free. No additional data are available.

\section{Disclosure}

We declare that we have no financial and personal relationships with other people or organizations that can inappropriately influence our work, there is no professional or other personal interest of any nature or kind in any product, service and/or company that could be construed as influencing the position presented in, or the review of, the manuscript entitled.

\section{References}

1. Weber T, Wassertheurer S, O'Rourke MF, et al. Pulsatile hemodynamics in patients with exertional dyspnea: potentially of value in the diagnostic evaluation of suspected heart failure with preserved ejection fraction. J Am Coll Cardiol. 2013;61:1874-1883. doi:10.1016/j. jacc.2013.02.013

2. Shah SJ, Kitzman DW, Borlaug BA, et al. Phenotype-specific treatment of heart failure with preserved ejection fraction: a multiorgan roadmap. Circulation. 2016;134:73-90. doi:10.1161/ CIRCULATIONAHA.116.021884

3. Upadhya B, Kitzman DW. Heart failure with preserved ejection fraction: new approaches to diagnosis and management. Clin Cardiol. 2020;43:145-155. doi:10.1002/clc.23321

4. Pandey A, Khera R, Park B, et al. Relative impairments in hemodynamic exercise reserve parameters in Heart Failure with preserved ejection fraction: a Study-Level Pooled Analysis. JACC Heart Fail. 2018;6:117-126. doi:10.1016/j.jchf.2017.10.014
5. Schneider SIDR, Silvello D, Martinelli NC, et al. Plasma levels of microRNA-21, -126 and $-423-5 p$ alter during clinical improvement and are associated with the prognosis of acute heart failure. Mol Med Rep. 2018;17:4736-4746. doi:10.3892/mmr.2018.8428

6. Wang X, Lian Y, Wen X, et al. Expression of miR-126 and its potential function in coronary artery disease. Afr Health Sci. 2017; 17:474-480. doi:10.4314/ahs.v17i2.22

7. Ghorbanzadeh V, Mohammadi MD, Dariushnejad H. Cardioprotective effect of crocin combined with voluntary exercise in rat: role of Mir-126 and Mir-210 in heart angiogenesis. Arq Bras Cardiol. 2017;109:54-62. doi:10.5935/abc.20170087

8. Ma C, Wang J, Liu H, et al. Moderate exercise enhances endothelial progenitor cell exosomes release and function. Med Sci Sports Exerc. 2018;50(10):2024-2032. doi:10.1249/MSS.0000000000001672

9. Jones B, Zhou Y, Goodwin AJ, et al. Application of deacetylated Poly-N-Acetyl glucosamine nanoparticles for the delivery of miR-126 for the treatment of cecal ligation and puncture-induced sepsis. Inflammation. 2019;42:170-184. doi:10.1007/s10753-018-0882-8

10. Wesley JT, Siddhartha SA, Mark JH. Pathophysiology of exercise intolerance and its treatment with exercise-based cardiac rehabilitation in heart failure with preserved ejection fraction. $J$ Cardiopulm Rehabil Prev. 2020;40:9-16. doi:10.1097/HCR.0000000000000481

11. Naderi R, Mohaddes G, Mohammadi M, et al. The effect of garlic and voluntary exercise on cardiac angiogenesis in diabetes: the role of MiR-126 and MiR-210. Arq Bras Cardiol. 2019;112:154-162. doi:10.5935/abc.20190002

12. DeRosa S, Eposito F, Carella C, et al. Transcoronary concentration gradients of circulating microRNAs in heart failure. Eur J Heart Fail. 2018;20:1000-1010. doi:10.1002/ejhf.1119

13. Amr KS, Abdelmawgoud H, Ali ZY, et al. Potential value of circulating microRNA-126 and microRNA-210 as biomarkers for type 2 diabetes with coronary artery disease. $\mathrm{Br} J$ Biomed Sci. 2018;75:82-87. doi:10.1080/09674845.2017.1402404

14. Wei XJ, Han M, Yang FY, et al. Biological significance of miR-126 expression in atrial fibrillation and heart failure. Braz J Med Biol. 2015;48:983-989. doi:10.1590/1414-431X20154590

15. Chen L, Wang J, Wang B, et al. MiR-126 inhibits vascular endothelial cell apoptosis through targeting PI3K/Akt signaling. Ann Hematol. 2016;95:365-374. doi:10.1007/s00277-015-2567-9

16. Chistiakov A, Orekhov A, Bobryshev YV. The role of miR-126 in embryonic angiogenesis, adult vascular homeostasis, and vascular repair and its alterations in atherosclerotic disease. $J$ Mol Cell Cardiol. 2016;97:47-55. doi:10.1016/j.yjmcc.2016.05.007

17. Khanaghaei M, Tourkianvalashani F, Hekmatimoghaddam S, et al. Circulating miR-126 and miR-499 reflect progression of cardiovascular disease; correlations with uric acid and ejection fraction. Heart Int. 2016;11:e1-e9. doi:10.5301/heartint.5000226

18. Fukushima Y, Nakanishi M, Nonogi H, et al. Assessment of plasma miRNAs in congestive heart failure. Circ J. 2011;75(2):336-340. doi:10.1253/circj.cj-10-0457

19. Dastah S, Tofighia A, Azar JT, et al. The role of miR-126 in embryonic angiogenesis, adult vascular homeostasis, and vascular repair and its alterations in atherosclerotic disease. Gene Rep. 2020;21(12):100914. doi:10.1016/j.genrep.2020.100914

20. Fish JE, Santoro MM, Morton SU, et al. miR-126 regulates angiogenic signaling and vascular integrity. Dev Cell. 2008;15(2):272-284. doi:10.1016/j.devcel.2008.07.008

21. Wang S, Aurora AB, Johnson BA, et al. The endothelial-specific microRNA miR-126 governs vascular integrity and angiogenesis. Dev Cell. 2008;15:261-271. doi:10.1016/j.devcel.2008.07.002

22. Da silva ND Jr, Fernandes T, Soci UP, et al. Swimming training in rats increases cardiac MicroRNA-126 expression and angiogenesis. Med Sci Sports Exerc. 2012;44(8):1453-1462. doi:10.1249/MSS.0b013e31824e8a36

23. Hijmans JG, Diehl KJ, Bammert TD, et al. Association between hypertension and circulating vascular-related microRNAs. J Hum Hypertens. 2018;32:440-447. doi:10.1038/s41371-018-0061-2 


\section{Publish your work in this journal}

The International Journal of General Medicine is an international, peer-reviewed open-access journal that focuses on general and internal medicine, pathogenesis, epidemiology, diagnosis, monitoring and treatment protocols. The journal is characterized by the rapid reporting of reviews, original research and clinical studies across all disease areas. The manuscript management system is completely online and includes a very quick and fair peer-review system, which is all easy to use. Visit http://www.dovepress.com/ testimonials.php to read real quotes from published authors. 